\title{
A comparative study on the effect of disks geometrical features on the drag torque of a wet friction clutch
}

\author{
Nikolaos Rogkas ${ }^{1, *}$, Despoina Almpani ${ }^{1}$, Georgios Vasileiou ${ }^{1}$, Eustratios Tsolakis ${ }^{1}$, Christos Vakouftsis $^{1}, P$. Zalimidis $^{2}$, \\ and Vasilios Spitas ${ }^{1}$ \\ ${ }^{1}$ Machine Design Laboratory, School of Mechanical Engineering, National Technical University of Athens, 9 Heroon Polytechniou Str., \\ 15780 Zografou, Greece \\ ${ }^{2}$ School of Pedagogical and Technological Education Department of Mechanical Engineering Educators, Iraklio Attikis, 14121 Athens, \\ Greece
}

\begin{abstract}
In recent years, the need for highly efficient automatic and semiautomatic powertrains has led to the wide use of wet friction clutches as power transmission systems. While wet friction clutches surpass their counterparts dry-friction clutches in the engagement process as they provide higher efficient torque and smoother torque and speed transmission, yet they fall behind in their disengaged state where the clutch acts as a power loss to the system due to the drag torque developed on the disks from fluid's viscous shear stresses. Shear stresses are developed due to the relative speed difference of coaxial disks distanced at a prescribed axial clearance and rotating independently, yielding to a torsional Couette shear flow. This matter has been investigated thoroughly in the literature with experimental and numerical approaches for the case of flat disks admitting the complex flow pattern, which is augmented even more from the presence of geometrical features (grooves) on the surface of the disks. It becomes clear that the efficient calculation and understanding of drag torque can lead to its minimization and therefore to further establishing the use of wet clutches. In this work the effect of various disks geometrical features on the drag torque of a wet clutch is examined. The results are obtained via FEA (Finite Element Analysis) using the commercial software ANSYS.
\end{abstract}

\section{Introduction}

In this century the reduction of environmental pollution associated with automobiles emissions is an urgent need for the automotive industry. Emissions of automobiles driven by Internal Combustion Engines (ICEs) with combustion of hydrocarbons, working on the sparkignition or diesel principle, are responsible both for the planet temperature increase (GHG emissions) as well as for the people health deterioration (PM emissions). In 2012, almost the entire (99.8\%) global vehicle stock was still based on internal combustion engine vehicles (ICEVs) using petroleum-based fuels [1], while the estimated total vehicles in 2050 is calculated to exceed 2 billion [2]. For the case of EU, it is notable that while the total $\mathrm{CO}_{2}$ (most relevant greenhouse gas) emissions were reduced by almost $12 \%$ during 1995-2010, the transport sector $\mathrm{CO}_{2}$ emissions were increased by almost $12 \%$ during the same period. In 2019 the transport-related emissions $\mathrm{CO}_{2}$ were surpassing $30 \%$ of the EU's total $\mathrm{CO}_{2}$ emissions with passenger cars standing out as a major polluter, accounting for $60.7 \%$ of total $\mathrm{CO} 2$ emissions [3].

Therefore, reducing GHG and PM emissions as well as fuel usage, led the automotive industry to promote new powertrain technologies and alternative transmission layouts. By optimizing the power transmission system, the engine will operate with better fuel efficiency, which contributes directly to the reduction of GHG and PM emissions and improved fuel usage. Novel transmission layouts such as dual-clutch transmissions (DCTs) and planetary automatic transmissions (ATs) have continuously emerged and embedded in demanding powertrains of ICEVs and Hybrid Electric Vehicles (HEVs). ATs layouts offer increased number of speeds $(7,8$, and 10 -speed layouts have been industrialized) aiming to improved drivability and fuel economy, while DCTs schemes integrate the great mechanical efficiency and manufacturability of manual transmissions (MTs) along with the capability of the power-shift module offered initially by ATs. [4].

Multidisk wet-friction clutches are incorporated in demanding powertrains of automatic and semi-automatic transmissions (DCTs, ATs, Limited Slip Differential) because they offer intrinsically more efficient cooling and less wear than their dry-friction counterparts and ensure high efficiency and smoothness during gearshift. Wet clutch assemblies act as fluidic power-control actuators (clutch/brake operation) and are featured by two main operations: engagement/disengagement $[5,6]$ phase and disengaged state $[7,8]$. The principle of their operation is the development of a torsional-type Couette

* Corresponding author: nrogkas@mail.ntua.gr 
shear flow between two coaxial discs rotating at different speeds. During the engagement/disengagement phase, the disks are moving axially, towards or away from each other, while during the disengaged state they are assumed to be fixed axially, separated by the fluid film. The operational regime of a wet clutch is rendered by various thermoelastohydrodynamic phenomena, including hydrodynamic, mixed and boundary lubrication with complex flow patterns and instabilities [9], heat transfer via conduction and convection [10, 11], aeration [12], as well as solid discs mechanics and diversification of the system dynamics over time due to changes in automatic transmission fluid (ATF) properties [13, 14]. Research on wet clutch engagement phase is ever growing since this phase characterizes the efficiency of the device. However, a side-effect of the wet friction clutches is that they absorb power continuously in the disengaged state due to the drag torque developed on the disks due to the viscosity of the fluid. According to [15] almost 5-6\% of total vehicle losses correspond to losses in the drivetrain including drag torque losses. Therefore, the evaluation and reduction of drag torque constitutes an active research hotspot for widespread of the wet-clutch technology in the automotive industry.

The evaluation of wet clutch drag torque conducted in literature, is usually followed by the drag torque speed graph, which indicates the influence of rotating disks speed difference on the magnitude of drag torque. In the low-speed regime, the correlation converges to a linear form, until a critical speed value, which generates the peak drag torque. After surpassing the critical speed which usually is located between 650$1000 \mathrm{rpm}$, drag torque drops instantly since air is inserted between the discs, reducing the apparent viscosity [12]. Finally, as the speed difference continuous to rise, approaching values of 5000rpm, unstable vibration characteristics in high circumferential speed regimes produce plate wobbling and mechanical contact, leading to impact and wear phenomena [13, 14].

The transition from one-phase, automatic transmission fluid (ATF) flow to two-phase, ATF-air flow, conducts an active open issue related to the wet clutch technology. The physics of the disengaged state, which resemble the Von Karman's swirling flow problem, along with the absence of flexible disks displacements such in the case of engagement phase, suggest the experimental approaches as the most comprehensive and trust-worthy attempt in order to yield characteristics of the transition and therefore various related studies are available [7, 16]. However, the associated experimental costs and the need to obtain generalized results, have led to the formulation of analytical or computational mathematical models.]

An approximate expression of drag torque (T) [17] which is valid at low speeds is:

$$
\mathrm{T} \propto \mathrm{rA}\left(\frac{\mu \mathrm{v}}{\mathrm{h}}\right)
$$

where (r) is the reference radius, (A) the surface area, $(\mu)$ the dynamic viscosity, ( $v$ ) the speed and (h) the film thickness. Based on this relation, the possible control techniques applicable for reduction of drag torque in low-speed regimes are: a) reduction of net surface encountering fluids viscous shear stresses, via introduction of geometrical features on the friction disk surface (grooves), b) increase of nominal film thickness, c) control of viscosity of ATF, d) control of inflow rate (Q) which is associated with the velocity (v).

This paper deals with the reduction of drag toque via the introduction of grooves on the surface of the friction disk. Two different grooves patterns (radial and newly introduced arcbow grooves) are compared against the case of non-grooved flat disks. Besides the effect of the groove shape, the effect of its size (width, depth) is also evaluated.

\section{Fluid film modeling}

\subsection{Governing equations}

The fluid film model presented in Fig. 1, consists of two annulus disks (top, bottom) rotating with speed $\left(\omega_{1}\right)$ and $\left(\omega_{2}\right)$ respectively, separated by a fluid film of thickness (h). Fluid enters the gap from the inner radius $\left(r_{i n}\right)$ and exits from the outer radius $\left(\mathrm{r}_{\text {out }}\right)$ where the pressure equals the ambient.

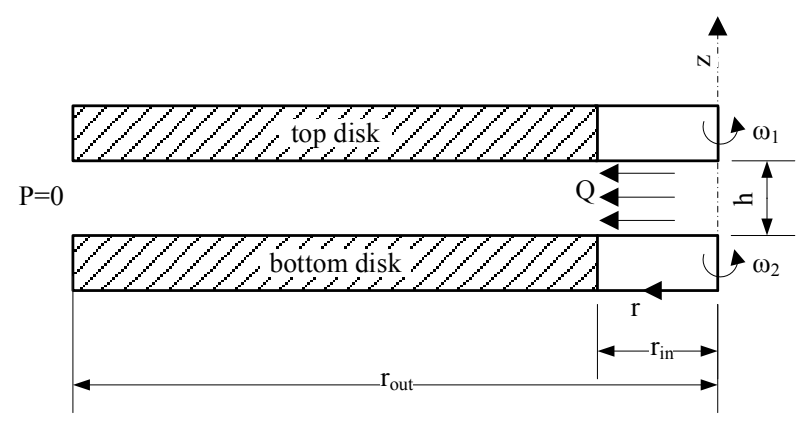

Fig. 1. Model.

The model of Fig. 1 is used by the researchers in order to solve truncated forms of Navier-Stokes equations, yielding the influence of the inflow rate $(\mathrm{Q})$ on the developed fluid pressure $(\mathrm{P})$. The flow is considered steady and isothermal and the ATF is assumed incompressible. The velocity vector is $\mathbf{u}=\left[\begin{array}{lll}u_{\mathrm{r}} & \mathrm{u}_{\vartheta} & \mathrm{u}_{\mathrm{z}}\end{array}\right]^{\mathrm{T}}$ and the continuity and Navier-Stokes equations are written in the inertial reference frame:

$$
\begin{gathered}
\nabla \cdot \mathbf{u}=0 \\
\mathbf{u} \cdot \nabla \mathbf{u}=-\frac{1}{\rho} \nabla \mathbf{P}+v \nabla^{2} \mathbf{u}
\end{gathered}
$$

According to [18], Eq. (2) may be simplified to the following form:

$$
-\frac{\mathrm{u}_{\theta}^{2}}{\mathrm{r}}=-\frac{1}{\rho} \frac{\partial \mathrm{P}}{\partial \mathrm{r}}+\frac{\mu}{\rho} \frac{\partial^{2} \mathrm{u}_{\mathrm{r}}}{\partial \mathrm{z}^{2}}
$$




$$
\mathrm{v}_{\mathrm{r}} \frac{\partial \mathrm{v}_{\theta}}{\partial \mathrm{r}}+\frac{\mathrm{v}_{\mathrm{r}} \mathrm{v}_{\theta}}{\mathrm{r}}=\frac{\mu}{\rho} \frac{\partial^{2} \mathrm{v}_{\theta}}{\partial \mathrm{z}^{2}}
$$

under the boundary conditions:

$$
\begin{gathered}
P\left(\mathrm{r}_{\text {out }}\right)=\mathrm{P}_{\mathrm{a}}=0 \\
\mathrm{u}_{\mathrm{z}}(\mathrm{r}, \mathrm{h})=0 \text { and } \mathrm{u}_{\mathrm{z}}(\mathrm{r}, 0)=0 \\
\mathrm{u}_{\mathrm{r}}(\mathrm{r}, \mathrm{h})=0 \text { and } \mathrm{u}_{\mathrm{r}}(\mathrm{r}, 0)=0 \\
\mathrm{u}_{\theta}(\mathrm{r}, \mathrm{h})=\omega_{1} \mathrm{r} \text { and } \mathrm{u}_{\theta}(\mathrm{r}, \mathrm{h})=\omega_{2} \mathrm{r}
\end{gathered}
$$

The coupled system of Eqs. (3), (4) is solved analytically under the boundary conditions of Eqs. (5-8) in [18] and the pressure distribution versus the radius is obtained. It is concluded that the developed pressure may be either positive or negative, depending on whether or not the inflow rate is larger than the flow rate induced by the inertial force. The following condition is derived:

$$
\mathrm{Q} \leq \frac{\rho \mathrm{h}^{3} \pi\left(\mathrm{r}_{\text {out }}^{2}-\mathrm{r}^{2}\right)\left(\omega_{2}^{2}-\omega_{2} \Delta \omega+\frac{3}{10} \Delta \omega^{2}\right)}{12 \mu \ln \frac{\mathrm{r}_{\text {out }}}{\mathrm{r}}}
$$

In this study, the viscosity and density values are calculated from the equations [12]:

$$
\begin{gathered}
\mu=\alpha \mathrm{e}^{\frac{\mathrm{b}}{\mathrm{T}+\mathrm{c}}}, \mathrm{T} \text { in }[\square] \\
\mathrm{a}=6.27 \cdot 10^{-5} \mathrm{Pas}, \mathrm{b}=1005.2 \square, \mathrm{c}=124.95 \square \\
\rho=-0.612 \cdot \mathrm{T}+854,18\left[\frac{\mathrm{kg}}{\mathrm{m}^{3}}\right], \mathrm{T} \text { in }[\square]
\end{gathered}
$$

Where the values of Table 1 are used. The inflow rate (Q) satisfies Eq. 9.

Table 1. Parameters.

\begin{tabular}{cccc}
\hline Symbol & Description & Value & Unit \\
\hline $\mathrm{r}_{\text {out }}$ & Outer radius & 70 & $\mathrm{~mm}$ \\
$\mathrm{r}_{\text {in }}$ & Inner radius & 50 & $\mathrm{~mm}$ \\
$\mathrm{~h}$ & Film thickness & 0.25 & $\mathrm{~mm}$ \\
$\mathrm{Q}$ & Inflow rate & 3.58 & $\mathrm{lpm}$ \\
$\omega_{1}$ & top disk speed. & $100-700$ & $\mathrm{rpm}$ \\
$\omega_{2}$ & bot. disk speed & 0 & $\mathrm{rpm}$ \\
$\mathrm{T}$ & Temperature & 26.5 & $\square$ \\
$\mu$ & Dynamic viscosity & 0.0478 & $\mathrm{~Pa} \mathrm{~s}$ \\
$\rho$ & Density & 837.9 & $\mathrm{Kg} / \mathrm{m} 3$ \\
\hline
\end{tabular}

\subsection{Grooves geometry}

The examined grooves patterns are presented in Figs. 24.

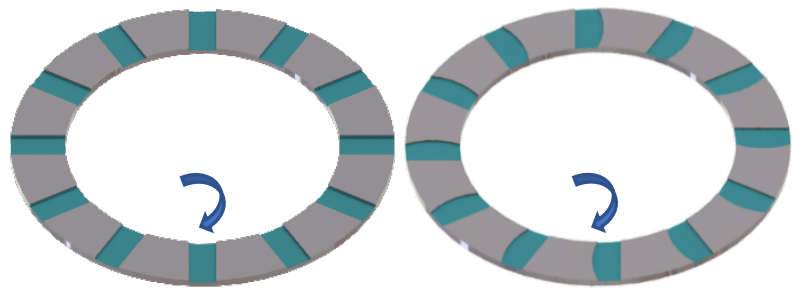

Fig. 2. Left: Radial groove pattern, right: Arcbow groove pattern.

The use of radial grooves in wet clutch application is common since they reduce the developed pressure during the engagement phase and the drag torque during the disengaged state via the decrease of net surface encountering fluids viscous shear stresses. Nonetheless, the potential of radial grooves design in terms of increasing the engagement hydrodynamic torque is limited since their vertical surface (where normal stresses are applied during engagement) length is restricted by the term $\left(\mathrm{r}_{\text {out }}-\mathrm{r}_{\text {in }}\right)$. The newly introduced arcbow grooves aim to elongate the vertical surface where normal stresses are exerted.

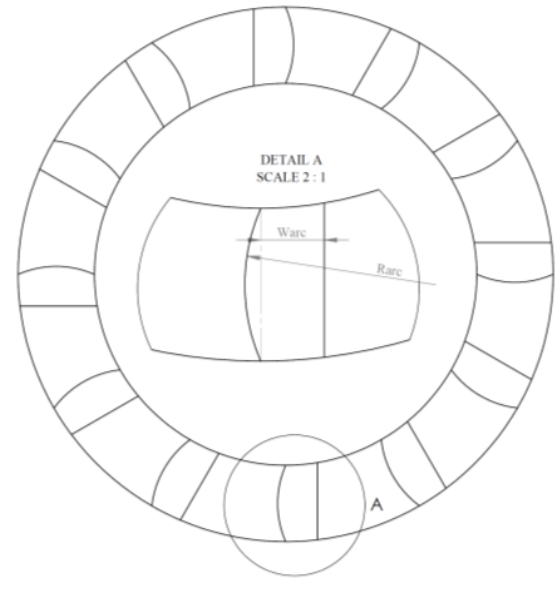

Fig. 3 Radial grooves pattern: geometry and basic dimensions. The number of grooves equals to 12 .

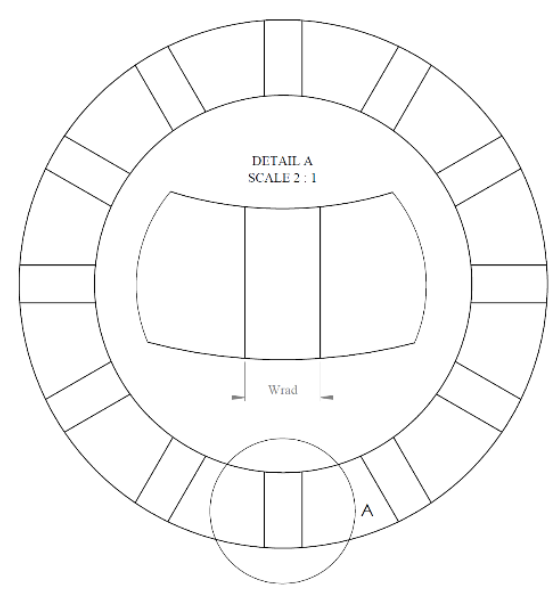

Fig. 4 Arcbow grooves pattern: geometry and basic dimensions. The number of grooves equals to 12 .

The parameters of the grooves geometry are given in Table 2, where (d) is the depth of the groove. 
Table 2. Grooves parameters.

\begin{tabular}{cc|ccc}
\hline \multicolumn{3}{c|}{ Radial groove } & \multicolumn{3}{c}{ Arcbow groove } \\
\hline $\mathrm{w}(\mathrm{mm})$ & $\mathrm{d}(\mathrm{mm})$ & $\mathrm{R}(\mathrm{mm})$ & $\mathrm{w}(\mathrm{mm})$ & $\mathrm{d}(\mathrm{mm})$ \\
5 & 0.5 & 13.5 & 5 & 0.5 \\
7.5 & 1 & 25 & 8 & 1 \\
10 & & & & \\
\hline
\end{tabular}

\section{CFD analysis}

\subsection{CFD model setup}

In order to compare the drag torque of the grooved disks of Fig. 2 against the drag torque of flat disks, a CFD analysis was conducted. The geometry of the model along with the boundary conditions (Eqs. 5-8) is shown in Fig. 1. The CFD environment used was ANSYS Fluent and the model was treated as 3D one. For both approaches a mesh independency study was carried out leading to mesh sizes of approximately 500,000 hexahedral elements for each geometry (Fig. 5, 6). The flow was considered steady for each value of top disk speed (100rpm, 300rpm, 500rpm and 1000rpm), laminar, and the residuals were set below $10^{-4}$ for all equations. SIMPLE algorithm was used for the couple of pressure and velocity, and second order spatial discretization scheme was used for the momentum conservation and pressure equations.

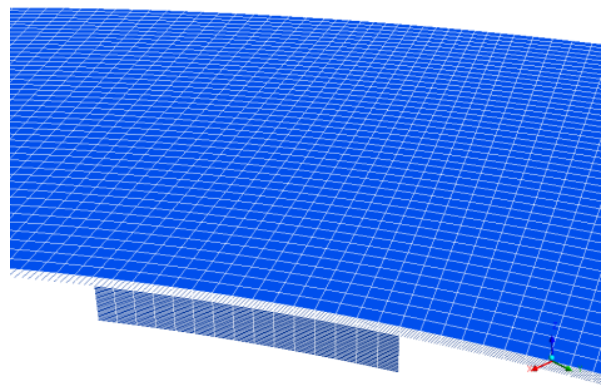

Fig. 5 Radial grooves model. Computational mesh details.

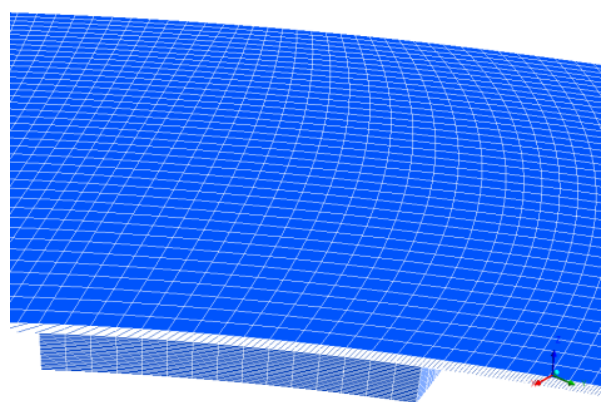

Fig. 6 Arcbow grooves model. Computational mesh details.
According to Eq. (9), the selected inflow rate $(3.58 \mathrm{lpm})$ prevents the event of reversed flow at the boundaries since the pressure distribution remains positive. However, the condition of Eq. (9) was derived from the solution of simplified forms of NS equation and thus considerable truncation errors may occur if the neglected terms of Eqs. (3-4) become important [19]. In addition, the presence of grooves in the geometry of the model, indicates another potential cause for the deviation between the results from the Eqs $(3,5)$ and the CFD model. For the needs of the study it was assumed that only ATF may re-enter the domain if reversed flow occurs.

\subsection{Results of CFD analysis}

For the presentation of the results, the non-dimensional drag torque coefficient is defined as:

$$
c_{m}=\frac{M}{\frac{1}{2} \rho v^{2} A L}
$$

where (M) is the drag torque, $A=\pi\left(r_{\text {out }}^{2}-r_{\text {in }}^{2}\right)$ is the area, $\mathrm{v}=\mathrm{r}_{\text {out }} \cdot \omega_{1}$ is the velocity and $\mathrm{L}=\mathrm{r}_{\text {out }}-\mathrm{r}_{\text {in }}$ is the length.

Fig. 7 presents the results of the drag torque coefficient $\left(c_{m}\right)$ for the case of flat disks. The linear relation between $\left(c_{m}\right)$ and $\left(\omega_{1}\right)$ is confirmed.

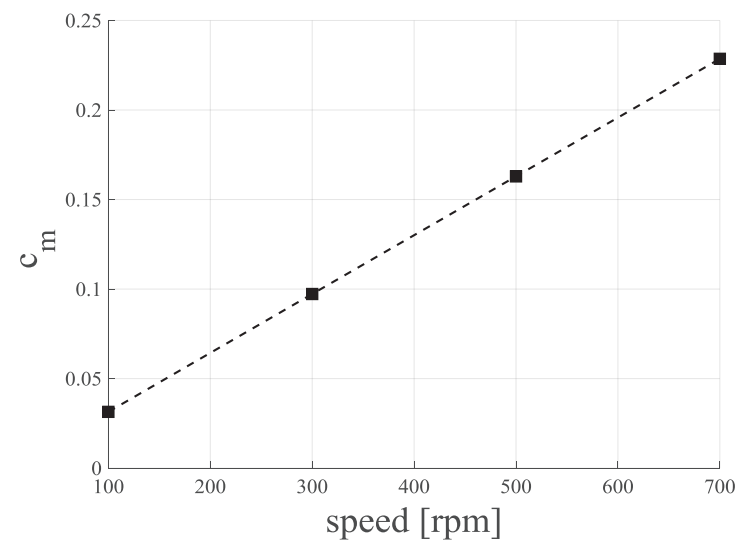

Fig. 7. Drag torque coefficient versus rotational speed of the top disk for non-grooved disks.

The comparison between the drag torque of flat nongrooved disks and drag torque of grooved disks is presented via the introduction of the percental drop of the drag-torque coefficient: $\left(\mathrm{c}_{\mathrm{m}_{\text {grooved }}}-\mathrm{c}_{\mathrm{m}_{\text {flat }}}\right) / \mathrm{c}_{\mathrm{m}_{\text {flat }}} \times$ $100 \%$

Fig. 8 illustrates the results of $\left(c_{m}\right)$ percental drop for the grooves configurations of Table 2 . 


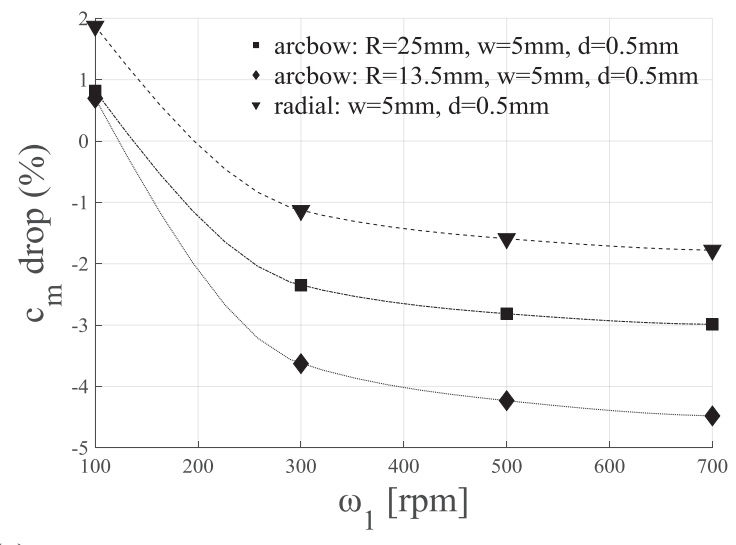

(a)

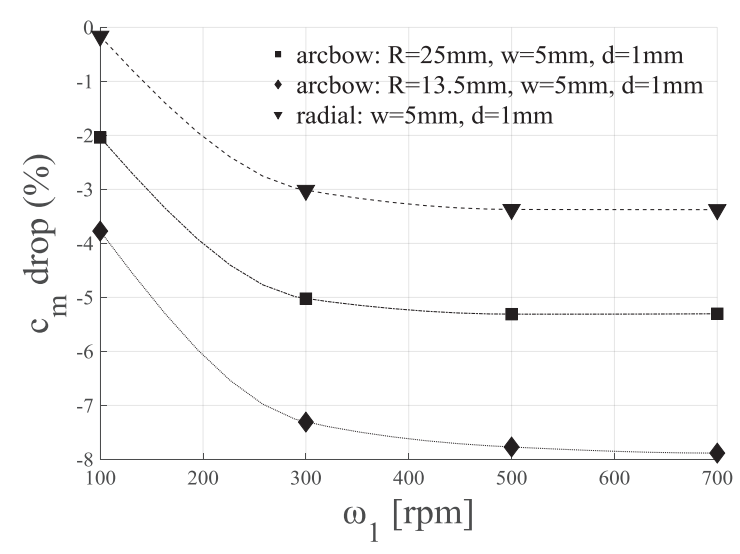

(b)

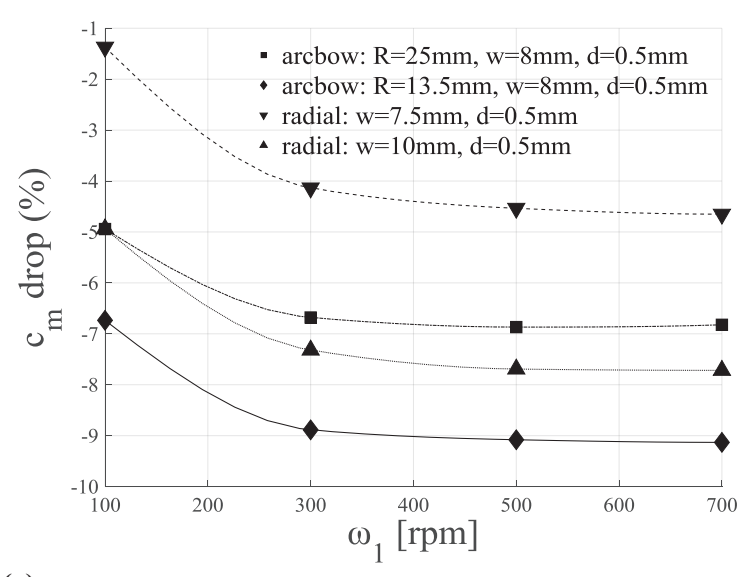

(c)

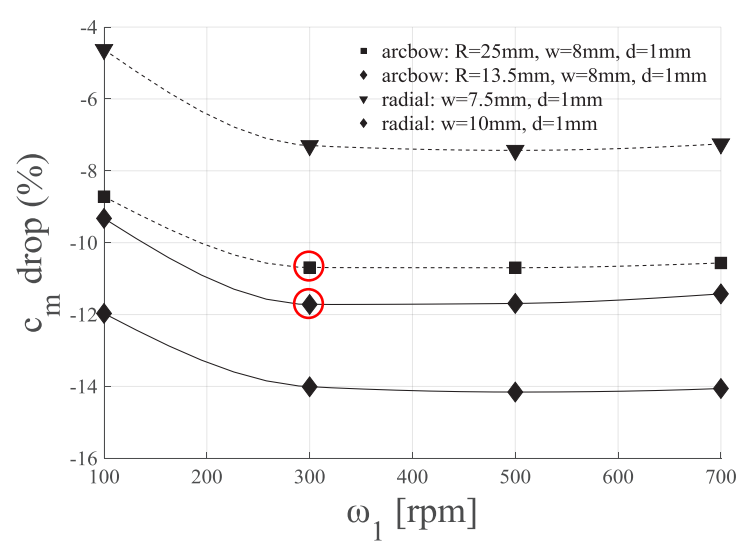

(d)
Fig. 8 (a)-(d). Percental drop of the drag-torque coefficient via the introduction of grooves on the friction disk. The grooves configurations corresponding to the parameters of Table 2 are evaluated.

Examining Fig. 8, it can be concluded that introduction of grooves on the surface of the friction disc may reduce considerably the drag torque coefficient. A reduction of $\left(\mathrm{c}_{\mathrm{m}}\right)$ up to $14 \%$ (Fig. 8-d) is detected under the presence of arcbow grooves with radius equal to $13.5 \mathrm{~mm}$. Generally, increasing the groove depth and area tends to reduce $\left(c_{m}\right)$ because the effective area on which fluid shear stresses are exerted is decreasing. This trend can be depicted in Fig. 9 where the magnitude of the zcomponent of the circumferential velocity gradient $\left(\partial \mathrm{u}_{\theta} / \partial \mathrm{z}\right)$ (which is associated with the drag torque) is presented in a contour plot for the two operating points shown in Fig 8-d. The magnitude of $\left(\partial \mathrm{u}_{\theta} / \partial \mathrm{z}\right)$ is decreased in the case of arcbow grooves.

Furthermore, the choice of arcbow grooves over radial grooves, implies a larger impact on the drag-torque reduction even for approximately equal groove width (w) and depth (d). The integration of an additional geometric parameter such as the arcbow radius $\left(\mathrm{R}_{\mathrm{arc}}\right.$ in Fig. 3) offers greater flexibility to the grooves design process, thus it is indicated for the satisfaction of additional design criteria such as the reduction of engagement force or the increase of engagement hydrodynamic torque (normal stresses will also be applied on the groove's walls which are extended).

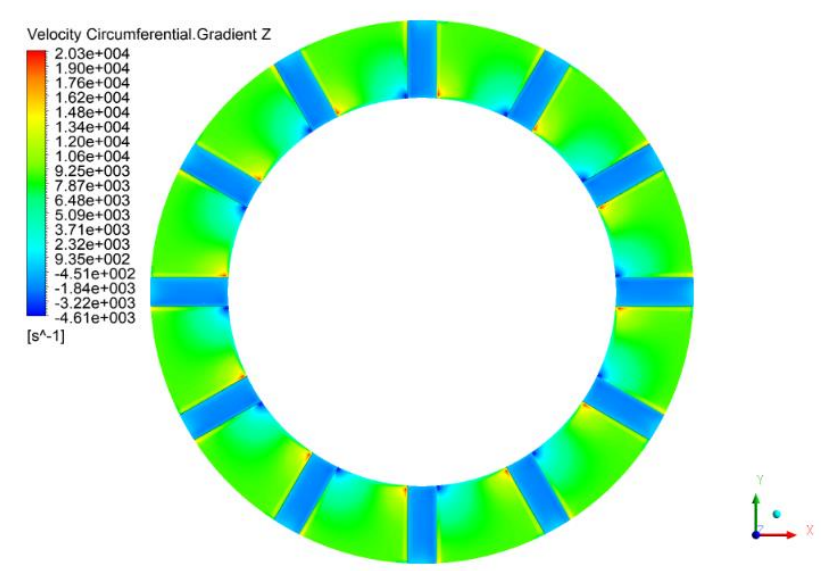




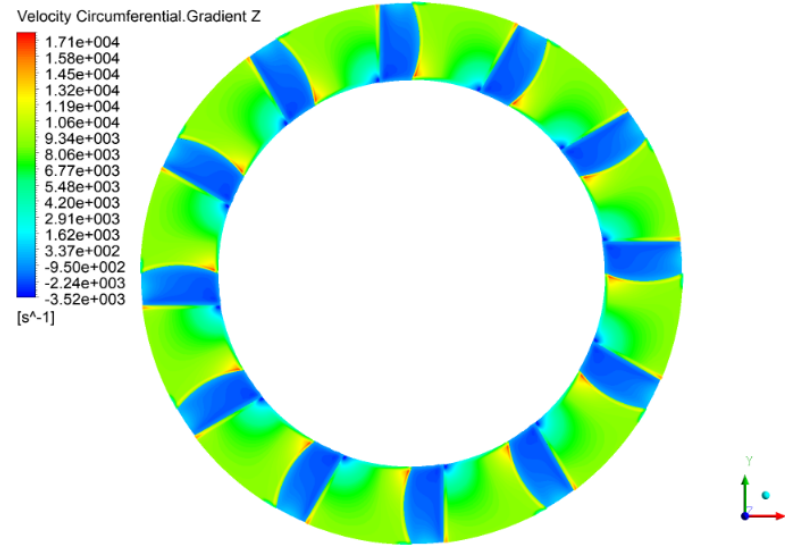

Fig. 9. Contour plot of the z-component of circumferential velocity gradient. Top: radial groove with $\mathrm{w}=7.5 \mathrm{~mm}, \mathrm{~d}=1 \mathrm{~mm}$ and bottom: arcbow groove with $\mathrm{R}=25 \mathrm{~mm}, \mathrm{w}=8 \mathrm{~mm}, \mathrm{~d}=1 \mathrm{~mm}$. Rotational speed equals to $300 \mathrm{rpm}$.

The velocity streamlines and vectors for the case of radial grooves with $\mathrm{w}=7.5 \mathrm{~mm}$ and $\mathrm{d}=1 \mathrm{~mm}$ (Fig. 9- Top) is presented in Fig. 11.
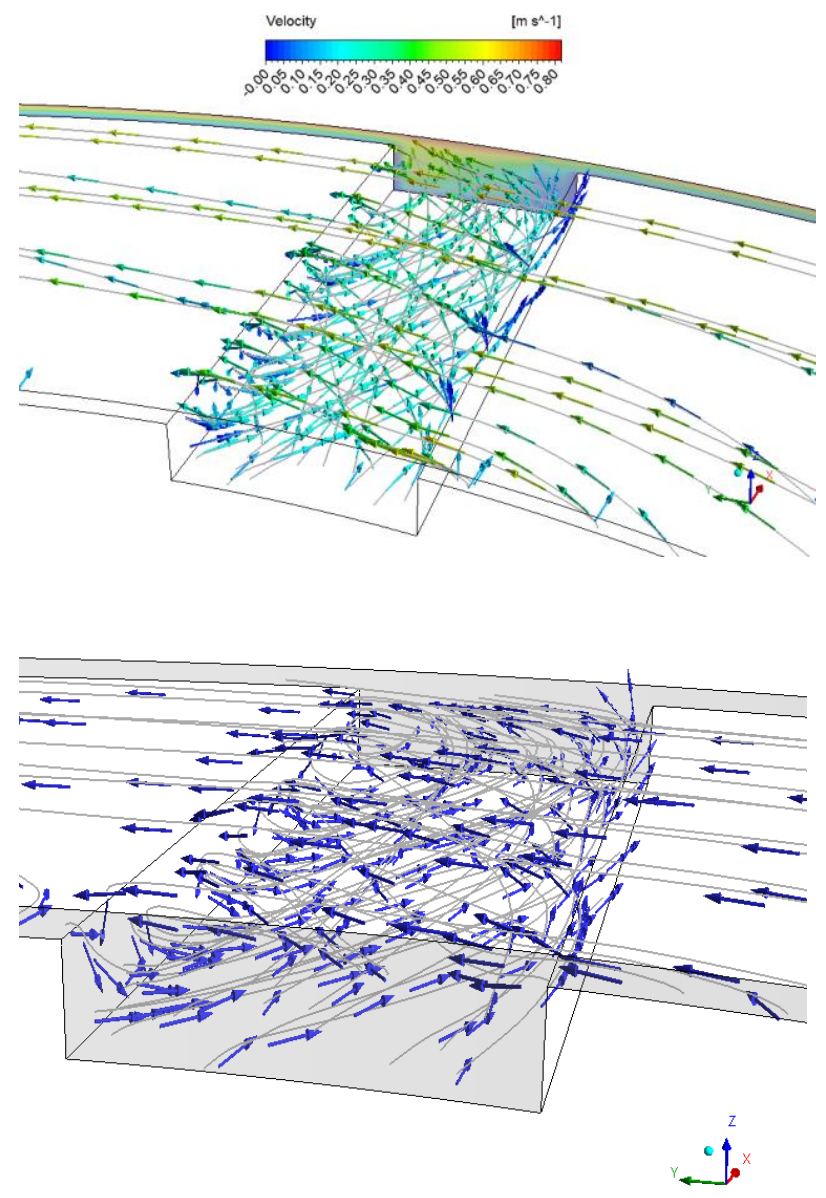

Fig. 10. Streamlines and velocity vectors close to a radial groove. Configuration: $\mathrm{w}=7.5 \mathrm{~mm}, \mathrm{~d}=1 \mathrm{~mm}, \omega_{1}=300 \mathrm{rpm}$. Top: overview, bottom: close-up inside the groove.

Fig. 10 shows that the circumferential component of the velocity field inside the groove is almost zero since the flow there is a combined radial-helical one. Regarding the radial component of the flow inside the groove, its contours are illustrated in Fig. 11. Negative values of radial velocity are observed near the outer radius region which indicates the event of reversed flow at the boundary.

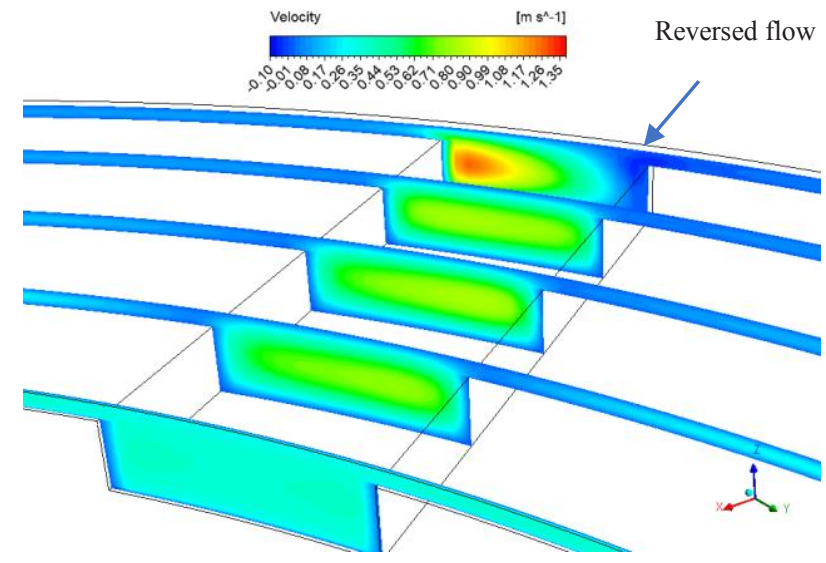

Fig. 11. Radial velocity contours inside a radial groove. Configuration: $\mathrm{w}=7.5 \mathrm{~mm}, \mathrm{~d}=1 \mathrm{~mm}, \omega_{1}=300 \mathrm{rpm}$.

\section{Conclusions}

In this study, a comparative study was conducted for the evaluation of the effect of different groove patterns on the drag torque of a wet friction clutch. The well-known radial grooves were compared against a newly introduced type (arcbow grooves) and results about the effect on the drag torque were obtained in the low-speed range (100 to $700 \mathrm{rpm})$. It is concluded that arcbow grooves may further reduce the drag torque magnitude by $2-3 \%$ compared to the traditional radial grooves.

This research is co-financed by Greece and the European Union (European Social Fund- ESF) through the Operational Programme «Human Resources Development, Education and Lifelong Learning» in the context of the project "Strengthening Human Resources Research Potential via Doctorate Research" (MIS-5000432), implemented by the State Scholarships Foundation (IKY)

\section{References}

1. Clean Energy Ministerial, (2013). Global EV Outlook, Paris, France.

2. M. Cames, \& E. Helmers. Environmental Sciences Europe, 25(1), 15 (2013)

3. Available in: https://www.europarl.europa.eu/news/en/headlines/s ociety/20190313STO31218/co2-emissions-fromcars-facts-and-figures-infographics

4. Xu, X., Dong, P., Liu, Y., \& Zhang, H. (2018). Progress in automotive transmission technology. Automotive Innovation, 1(3), 187-210.

5. Rogkas, N., Vasileiou, G., Tsolakis, E., Spitas, V., \& Zalimidis, P. (2019). Fast modelling and simulation of the dynamic behaviour of a wet 
multidisc clutch during the engagement phase. In MATEC Web of Conferences (Vol. 287, p. 01018). EDP Sciences.

6. Zhigang, Z., Xiaohui, S., \& Dong, G. (2016). Dynamic temperature rise mechanism and some controlling factors of wet clutch engagement. Mathematical Problems in Engineering, 2016.

7. S., Iqbal; F. Al-Bender; B. Pluymers; W. Desmet. Mathematical model and experimental evaluation of drag torque in disengaged wet clutches. ISRN Tribology 2013, vol. 2013, Article ID 206539, 16 pages.

8. Pan, H., \& Zhou, X. (2019, May). Simulation Research on the Drag Torque of Disengaged Wet Clutches. In 2019 IEEE 5th International Conference on Mechatronics System and Robots (ICMSR) (pp. 44-48). IEEE.

9. Zhao, J., Ma, B., \& Li, H. (2013, July). Investigation of thermoelastic instabilities of wet clutches. In 2013 IEEE International Symposium on Assembly and Manufacturing (ISAM) (pp. 69-72). IEEE.

10. AJen, T. C., \& Nemecek, D. J. (2008). Thermal analysis of a wet-disk clutch subjected to a constant energy engagement. International Journal of Heat and Mass Transfer, 51(7-8), 1757-1769.

11. Tatara, R. A., \& Payvar, P. (2002). Multiple engagement wet clutch heat transfer model. Numerical Heat Transfer: Part A: Applications, 42(3), 215-231.

12. Neupert, T., \& Bartel, D. (2019). High-resolution 3D CFD multiphase simulation of the flow and the drag torque of wet clutch discs considering free surfaces. Tribology International, 129, 283-296.

13. Zhang, L., Wei, C., Hu, J., \& Hu, Q. (2019). Influences of lubrication flow rates on critical speed of rub-impact at high circumferential velocities in No-Load multi-plate wet clutch. Tribology International, 140, 105847.

14. Hu, J., Hou, S., \& Wei, C. (2018). Drag torque modeling at high circumferential speed in open wet clutches considering plate wobble and mechanical contact. Tribology International, 124, 102-116.

15. Pahlovy, S. A., Mahmud, S. F., Kubota, M., Ogawa, M., \& Takakura, N. (2016). Prediction of drag torque in a disengaged wet clutch of automatic transmission by analytical modeling. Tribology Online, 11(2), 121-129.

16. Jibin, H., Zengxiong, P., \& Chao, W. (2012). Experimental research on drag torque for singleplate wet clutch. Journal of tribology, 134(1).

17. Fujii, Y.; Kapas, N.; Tseng, J. Clutch Wet in Encyclopedia of Automotive Engineering, John Wiley \& Sons, New York, 2014, pp .1-15.

18. Huang, J., Wei, J., \& Qiu, M. (2012). Laminar flow in the gap between two rotating parallel frictional plates in hydro-viscous drive. Chinese Journal of Mechanical Engineering, 25(1), 144-152.
19. Rogkas, N., Vakouftsis, C., Vasileiou, G., Manopoulos, C., \& Spitas, V. (2020). Nondimensional Characterization of the Operational Envelope of a Wet Friction Clutch. Computation, 8(1), 21. 